\title{
La spectroscopie confocale Raman en odontologie. De la recherche fondamentale aux applications cliniques
}

\section{RÉSUMÉ}

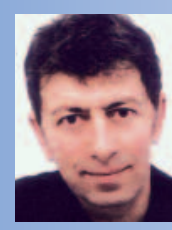

Bruno JACQUOT

MCU-PH,

Laboratoire Biosanté et Nanoscience, EA 4203,

UFR d'Odontologie,

Université Montpellier 1,

545 avenue du Prof.esseur J.-L. Viala,

34193 Montpellier Cedex 5.

Jean-Cédric DURAND

Ancien AHU,

Laboratoire Biosanté et Nanoscience, EA 4203,

UFR d'Odontologie,

Université Montpellier 1.

\section{Hugues FOUQUET}

Docteur en chirurgie dentaire,

Laboratoire Biosanté et Nanoscience, EA 4203,

UFR d'Odontologie,

Université Montpellier 1.

\section{Hamideh SALEHI}

Physicienne doctorante,

Laboratoire Biosanté et Nanoscience, EA 4203,

UFR d'Odontologie,

Université Montpellier 1.

\section{Perrine MARGUET-GAUDIN}

Ancienne AHU,

Laboratoire Biosanté et Nanoscience, EA 4203,

UFR d'Odontologie,

Université Montpellier 1.

\section{Bernard LEVALLOIS}

MCU-PH,

Laboratoire Biosanté et Nanoscience, EA 4203,

UFR d'Odontologie,

Université Montpellier 1.

\section{Frédéric CUISINIER}

PU-PH,

Laboratoire Biosanté et Nanoscience, EA 4203,

UFR d'Odontologie,

Université Montpellier 1.

Nous présentons un nouvel outil d'observation des surfaces, utilisé en recherche fondamentale, la spectroscopie Raman. Cette technique offre de nombreuses possibilités dans l'analyse et l'imagerie des tissus et des matériaux dentaires.

L'effet Raman a été découvert par Chandrashekhara Venkata Râman en 1928. Les applications sont très diverses en odontologie, mais I'utilisation majeure de la spectroscopie Raman en dentisterie concerne la mesure du degré de conversion soit directement au niveau du matériau pour les résines composites, soit à l'intérieur de la couche hybride pour les adhésifs.

La spectroscopie Raman, comme la spectroscopie infrarouge, est une méthode vibrationnelle, qui permet une approche directe du degré de conversion et une quantification de l'épaisseur de la couche inhibée par l'oxygène de l'air. Différentes applications sont illustrées et concernent la mesure du degré de conversion d'un adhésif amélodentinaire et la détermination de sa couche d'inhibition par l'oxygène de l'air.

- spectroscopie Raman

- recherche fondamentale

- degré de conversion

- résines composites

- couche inhibée par l'oxygène
AOS 2012;259:207-218

DOI: $10.1051 / a o s / 2012302$

(C) EDP Sciences 
$>$ L'exercice de la dentisterie, jour après jour, gagne en complexité et en difficulté. Grâce ou à cause d'Internet, les patients sont devenus des consommateurs de soins de santé, avec un regard critique sur nos modes de diagnostic et de thérapeutique. Or, ces thérapeutiques évoluent très rapidement au fur et à mesure des progrès techniques. Ces différents facteurs pèsent lourdement sur la prise de décisions cliniques et nécessitent, pour le praticien, de disposer d'informations pertinentes et de mises à jour régulières [1].

Il devient impossible d'utiliser uniquement les connaissances acquises au cours de nos études universitaires et la nécessité d'un changement de notre mode de formation devient indispensable.

Par ailleurs, nos orientations diagnostiques et thérapeutiques passent par un nouveau paradigme, la dentisterie fondée sur la preuve (Evidence Based Dentistry, EBD). Ce nouveau concept [2] est amplifié, comme pour nos patients, par la révolution Internet mais dans un sens positif et il nous permet de devenir un acteur majeur de notre formation. Ainsi, cette dentisterie fondée sur la preuve intègre obligatoirement la lecture critique d'articles (LCA). Une épreuve de LCA a été introduite à l'examen classant national de médecine en 2009 avec un impact progressif sur la note finale de l'examen. Ainsi, ce que tout praticien finissait par pratiquer spontanément dans sa formation continue au quotidien, de façon plus ou moins efficace, se trouve formalisé dans un enseignement dès le deuxième cycle des études médicales [3]. En odontologie, une épreuve de LCA a été intégrée au programme du concours d'internat pour cette année 2012 [4]. C'est un bouleversement considérable dans la pratique de la future formation continue odontologique.
La dentisterie de type EBD associée à la LCA réduit fortement l'écart entre la recherche et la pratique dentaire et fournit aux chirurgiensdentistes d'importants moyens pour accéder à cette recherche et en permettre son application clinique au fauteuil [1].

La recherche en odontologie est à la fois fondamentale et clinique. La recherche fondamentale est conduite principalement dans des laboratoires de recherche du CNRS, de I'INSERM et de I'Université. Cette recherche fondamentale cherche à décortiquer et à comprendre les systèmes biologiques et leur environnement, permettant ainsi la mise au point de biomatériaux innovants. Mais, les publications d'essais en laboratoire possèdent généralement un faible niveau de preuve. La recherche clinique se développe essentiellement au fauteuil et utilise les outils statistiques et épidémiologiques. Les publications de recherche clinique, au travers des essais contrôlés randomisés (Randomised Controlled Trial, $R C T$ ) et de leur synthèse par les méta-analyses, possèdent un niveau de preuve élevé.

Entre ces deux types de recherche s'intercale la recherche translationnelle. Cette recherche fait le lien entre la recherche fondamentale essentielle à tout progrès et la recherche clinique [5] se préoccupant du patient dans sa réalité complexe d'être humain, en développant les applications médicales des connaissances les plus récentes. Elle doit nécessairement se développer à proximité du patient. Le flux des connaissances doit être bi-directionnel, de la recherche vers le patient et du patient vers la recherche par l'intermédiaire du praticien.

La recherche translationnelle occupe aujourd'hui une position importante dans le paysage de la recherche biomédicale. En cancérologie, le Plan cancer recommande de soutenir la forma- 
tion à la recherche translationnelle en s'adressant également aux odontologistes [6]. La recherche translationnelle doit ainsi permettre la mise en œuvre optimale des connaissances les plus récentes dans la pratique odontologique. Dans ce contexte, le chirurgien-dentiste devient un acteur essentiel si ses connaissances sont mises à jour régulièrement par cet indispensable outil que devient la lecture critique d'articles.

Dans le cadre de ce besoin de corrélation entre la recherche fondamentale et ses applications cliniques, nous présentons un nouvel outil d'observation des surfaces utilisé en recherche fondamentale, la spectroscopie Raman.

Cette technique offre des possibilités extraordinaires dans l'analyse et l'imagerie des tissus et matériaux dentaires et permet de très nombreuses applications au laboratoire de recherche. De plus, sur le plan des applications cliniques, les chercheurs ont déjà commencé à transposer cette technique optique en un nouvel outil de diagnostic clinique utilisant diverses stratégies endoscopiques.

\section{La spectroscopie confocale Raman}

L'effet Raman a été découvert par Chandrashekhara Venkata Râman, physicien à I'université de Calcutta, qui obtient avec son équipe le premier spectre Raman en 1928. Pour démontrer ce nouveau type de diffusion de la lumière, les auteurs ont utilisé le rayonnement solaire focalisé sur un échantillon liquide (fig. 1) par un télescope de $18 \mathrm{~cm}$ d'ouverture et de $230 \mathrm{~cm}$ de focale et deux filtres colorés complémentaires [7]. La lumière violette du spectre solaire est isolée avec le filtre bleu-violet et passe à travers le liquide. La majorité de la lumière émergeant de l'échantillon liquide est de la même couleur que le faisceau incident, c'est la diffusion Rayleigh élastique (de même fréquence). Mais une toute petite partie de la lumière est d'une couleur différente, que Raman isole en utilisant le filtre jaune-vert placé entre l'observateur et l'échantillon, c'est la diffusion Raman non élastique (décalage en fréquence). Pour enregistrer le spectre Raman, des plaques photographiques étaient utilisées avec des temps de pause de plusieurs heures.
Ce montage originel rudimentaire s'est beaucoup perfectionné.

La première avancée technologique correspond à l'apparition du laser. Le laser produit, à

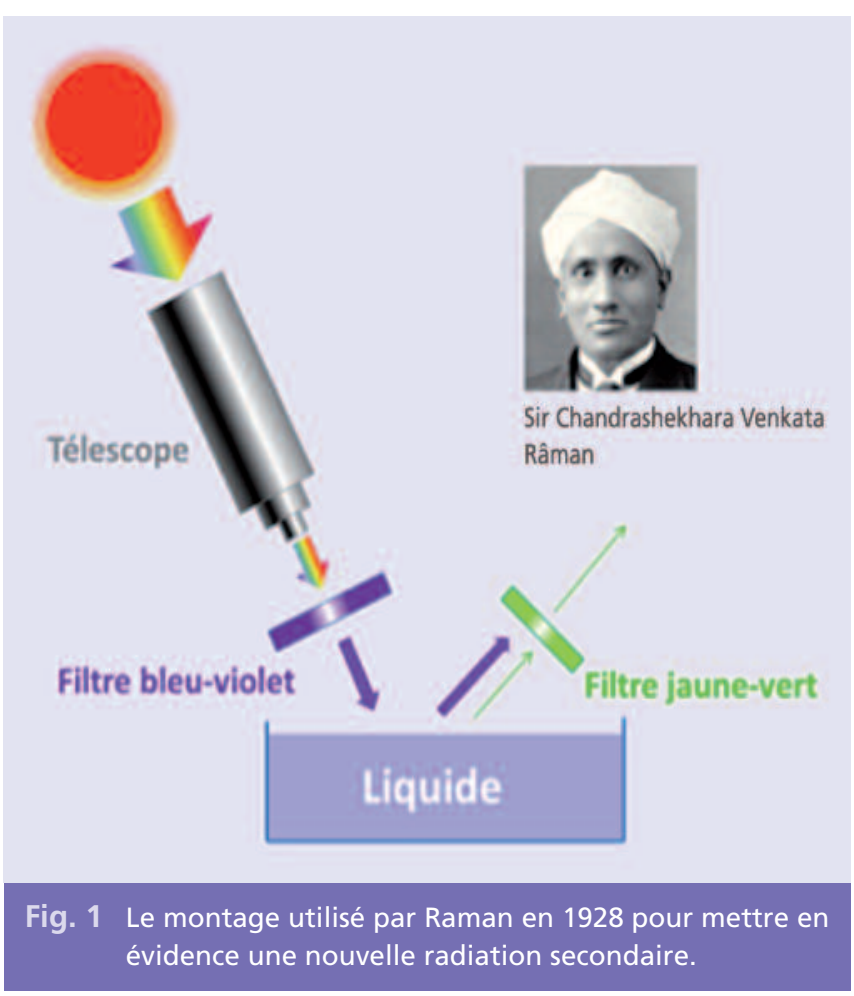


partir d'une source monochromatique, une lumière dont le rayonnement intense et concentré est émis à une longueur d'onde précise. L'intensité du rayonnement permet d'augmenter le nombre de photons diffusés et la fréquence étroite favorise la mesure du décalage en fréquence de la diffusion Raman.

La seconde avancée concerne l'apparition des capteurs photographiques de type CCD (Charge Coupled Device) dont l'efficacité est bien supérieure à la pellicule. On peut ainsi enregistrer un spectre Raman en moins d'une seconde. Enfin, le couplage avec un microscope confocal a fortement amélioré la résolution spatiale et permet également de réduire l'émission de fluorescence.

Ainsi la spectroscopie Raman est devenue beaucoup plus sophistiquée mais beaucoup plus facile à utiliser. Aujourd'hui, c'est la convergence de nombreux progrès en optique, microélectronique et informatique qui a révolutionné les performances de cette spectroscopie Raman.

L'effet Raman résulte de l'interaction des photons d'une source de lumière monochromatique avec les molécules ou le réseau cristallin de l'échantillon analysé. La majeure partie du faisceau incident est transmise, mais une petite partie de la lumière est diffusée. Pour cette diffusion, approximativement 1 sur 10000 photons est diffusé élastiquement et correspond à la diffusion Rayleigh. Occasionnellement, 1 photon est diffusé inélastiquement (1 sur 100000 000) avec une légère perte en énergie et correspond à la diffusion Raman. Ce processus de diffusion inélastique se produit en relation avec un changement de l'énergie vibrationnelle ou rotationnelle de la molécule ou du réseau cristallin. Ainsi, la spectroscopie Raman est définie comme une spectroscopie vibrationnelle comme la spectroscopie infrarouge.
Si on étudie un polymère, suite à l'impulsion laser, tous les atomes des différentes molécules vibrent avec une fréquence $v$ autour de leur position d'équilibre. Chaque noyau de la molécule vibre en phase mais avec une amplitude relative qui lui est propre. Certaines de ces vibrations entraînent des élongations des liaisons atomiques, on les appelle vibrations de valence ; d'autres induisent des déformations angulaires, on les appelle vibrations de déformation. Un mode de vibration sera observable en Raman si le mouvement correspondant s'accompagne d'une variation de la polarisabilité de la molécule.

Pour un polymère, du fait du nombre élevé d'atomes, il existe un nombre très important de modes fondamentaux vibrationnels. Mais, un grand nombre de ces modes ont presque la même fréquence. Ainsi, si plusieurs molécules contiennent la liaison $\mathrm{C}=\mathrm{C}$, le spectre Raman donnera une bande vers $1650 \mathrm{~cm}^{-1}$. Mais le nombre d'ondes précis dépend de l'environnement de la liaison étudiée. Par exemple, pour les polymères dentaires contenant le monomère Bis-GMA, la liaison $\mathrm{C}=\mathrm{C}$ du groupe vinyle possède un pic à $1640 \mathrm{~cm}^{-1}$ alors que la liaison $\mathrm{C}=\mathrm{C}$ du noyau aromatique possède un pic à $1610 \mathrm{~cm}^{-1}$. Un spectre Raman contient ainsi différents pics caractéristiques des liaisons chimiques constitutives de la molécule qui signent l'empreinte chimique du matériau analysé (fig. 2).

Tous les matériaux peuvent être analysés par spectroscopie Raman, quel que soit l'état sous lequel ils se présentent (solide, liquide ou gazeux).

De nombreuses informations chimiques, physiques et structurales peuvent être extraites d'un spectre Raman en fonction de la position des raies, de leur intensité, de leur décalage et de leur largeur. La position des raies renseigne sur les espèces chimiques en présence dans 
l'échantillon [8]. La largeur des pics fournit des informations sur la structure de l'échantillon. L'intensité d'un pic peut être reliée à la concentration de l'espèce [9] et à la température [10]. Le déplacement des pics est fonction de l'état de contrainte [11].

La spectroscopie Raman est ainsi hautement spécifique. De plus, cette technique optique se satisfait d'échantillons de très petite taille (de l'ordre du micromètre cube) et il est souvent possible de travailler sans préparation particulière de l'échantillon. L'enregistrement peut se

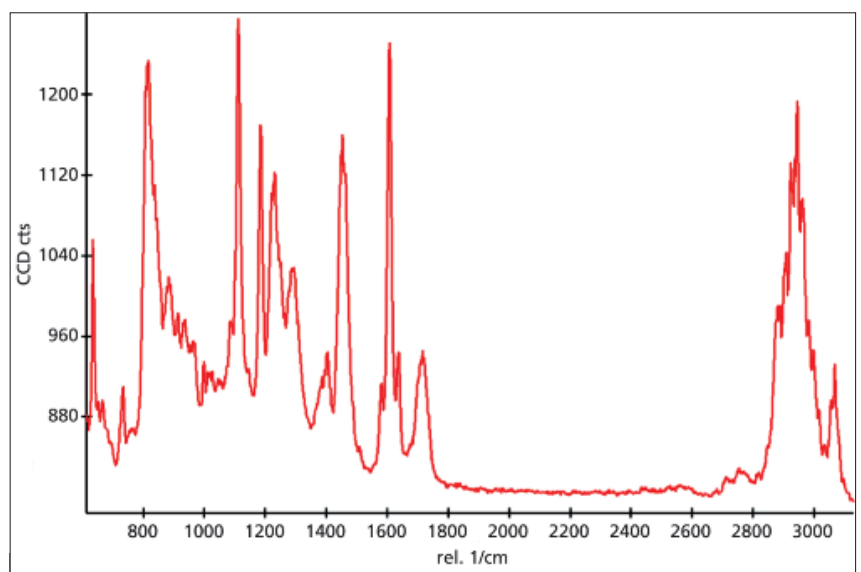

Fig. 2 Spectre Raman de Scotchbond MultiPurpose Bonding après polymérisation. réaliser à distance par l'intermédiaire de fibres optiques. Par ailleurs, on peut s'affranchir du problème de la fluorescence qui peut masquer le signal Raman, en utilisant la gamme actuelle des rayonnements qui va habituellement en odontologie de 532 nm à 1064 nm, le laser HeNe $632 \mathrm{~nm}$ étant le plus utilisé (fig. 3).

Cette technique est ainsi susceptible d'applications nombreuses et dans le domaine de l'imagerie, la résolution spatiale de l'ordre du micromètre permet des analyses topologiques ou cartographiques d'excellente qualité.

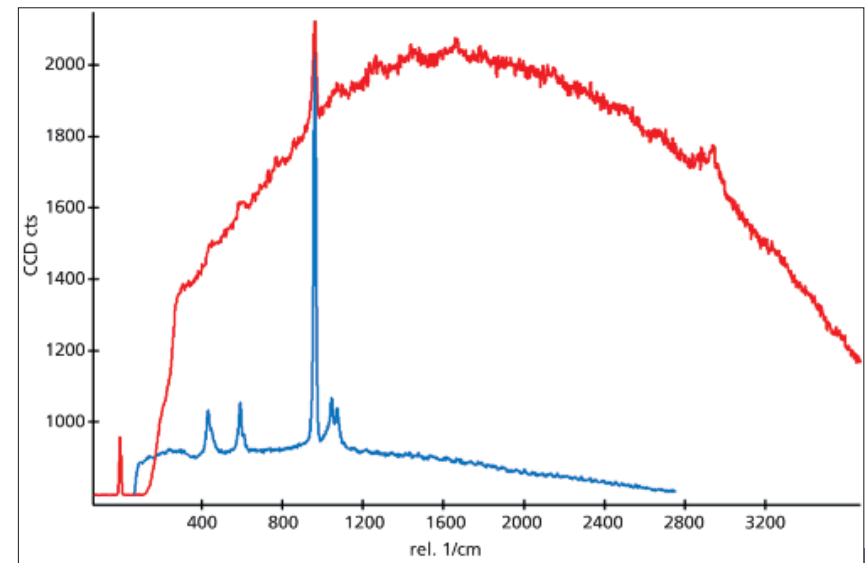

Fig. 3 Spectres Raman de l'émail humain avec le pic caractéristique des phosphates à $960 \mathrm{~cm}^{-1}$. Influence de la fluorescence sur les spectres obtenus en Spectroscopie Raman. En bleu, le spectre réalisé avec le laser $632 \mathrm{~nm}$ (faible fluorescence). En rouge, le spectre réalisé avec le laser $532 \mathrm{~nm}$ (forte fluorescence qui masque le signal Raman).

\section{L'équipement en spectroscopie confocale Raman}

Les éléments de base comprennent un laser associé à un microscope confocal et un spectromètre.

Le laser fournit une radiation monochromatique de fréquence stable et connue. Nous uti- lisons soit un laser He-Ne $632 \mathrm{~nm}$ soit un laser $\mathrm{Nd}-$ YAG $532 \mathrm{~nm}$, en fonction des échantillons testés.

Le microscope focalise le spot laser incident sur la surface de l'échantillon, via l'objectif approprié, 
puis dirige les photons diffusés vers le spectromètre.

Le processus Raman étant peu intense, un filtre holographique de type Notch est utilisé pour séparer le signal d'intérêt du signal Rayleigh beaucoup plus intense.

Les photons diffusés entrent dans le monochromateur par le trou confocal qui permet de localiser spatialement la lumière. Un miroir pris- matique dirige ensuite les photons sur un réseau holographique qui disperse les photons selon leur longueur d'onde.

Chaque radiation est ensuite focalisée par une lentille sur le détecteur le plus souvent de type CCD.

Le détecteur CCD permet de recueillir simultanément les informations spatiale et spectrale concernant l'échantillon analysé.

\section{La spectroscopie Raman en odontologie}

La première étude en odontologie s'est intéressée aux apatites de l'émail dentaire [12]. À la suite, la composition et la structure des différents tissus minéralisés ont été investiguées, ainsi que l'effet de différents traitements [13]. Pour la dentine, l'interface avec le Superbond (résine 4-META/MMA-TBB) a été initialement analysée [14] ; aujourd'hui la caractérisation de la pénétration des différents adhésifs amélo-dentinaires dans la dentine déminéralisée est couramment utilisée [15].

Pour l'émail, l'intérêt s'est d'abord porté sur I'utilisation de différents lasers comme le laser $\mathrm{CO}_{2}$ [16] et le laser Nd-YAG [17] ; aujourd'hui, la majorité des études s'appliquent à mettre en évidence la déminéralisation générée par I'utilisation du peroxyde d'hydrogène dans les techniques d'éclaircissement $[18,19]$.

Une autre application de la spectroscopie Raman concerne les céramiques dentaires, surtout la zircone Y-TZP.

En effet, la spectroscopie Raman permet de quantifier la proportion des phases monocli- nique et tétragonale dans la zircone et devient un outil performant pour l'analyse des transformations de phase lors de l'usinage ou de traitements de surface comme le sablage [20] mais également de la dégradation à basse température [21].

Mais I'utilisation majeure de la spectroscopie Raman en odontologie concerne la mesure du degré de conversion, soit directement au niveau du matériau pour les résines composites [22, 23], soit à l'intérieur de la couche hybride pour les adhésifs [24].

En effet, les propriétés physiques et mécaniques des composites dentaires photopolymérisables sont directement influencées par le degré de conversion atteint lors de la polymérisation [25].

L'adhérence des adhésifs amélo-dentinaires est également influencée par ce degré de conversion $[26,27]$.

La quantification de la couche d'inhibition générée par l'oxygène de l'air est également possible. 


\section{Application de la spectroscopie Raman à la mesure du degré de conversion des résines composites dentaires}

Pour mesurer le degré de conversion des résines composites, différentes techniques indirecte ou directe sont utilisées.

La micro-dureté permet d'évaluer de manière indirecte la profondeur de polymérisation des résines composites [28]. En général, des valeurs de dureté élevées sont un indicateur d'une polymérisation importante.

Un test de grattage, autre méthode indirecte, est également utilisé, la résine faiblement polymérisée de la profondeur de l'échantillon est brossée et l'épaisseur de polymérisation est mesurée [29].

Le microscope optique peut être utilisé pour détecter des changements dans l'indice de réfraction des résines et évaluer ainsi la profondeur de polymérisation [30].

Les méthodes vibrationnelles, la spectroscopie infrarouge et surtout la spectroscopie Raman permettent une approche directe du degré de conversion [31]. La spectroscopie Raman permet de mesurer l'épaisseur de la couche inhibée par l'oxygène de l'air mais aussi son degré de conversion [32]. La couche inhibée par l'oxygène est toujours présente en clinique lorsque I'adhésif ou le composite est polymérisé à l'air ambiant. Par contre un composite réalisé sous la pression d'une bande en mylar ne présente pas cette couche d'inhibition.

Le degré de conversion (DC) est obtenu en déterminant l'évolution de la concentration des doubles liaisons $C=C$ vinyliques (qui correspondent en Raman au pic $1640 \mathrm{~cm}^{-1}$ ) entre l'échantillon non polymérisé et l'échantillon polymérisé et en prenant la concentration en doubles liaisons $C=C$ aromatiques (qui correspondent en Raman au pic $1610 \mathrm{~cm}^{-1}$ ) comme référence, puisqu'elles ne participent pas à la réaction. La matrice polymère des composites utilisés dans les applications dentaires possède au moins deux types de monomères diméthacrylates dont au moins un contient deux noyaux aromatiques. Les adhésifs amélo-dentinaires ont des compositions variées avec le plus souvent un seul monomère diméthacrylate possédant deux noyaux aromatiques, mais certains adhésifs n'en possèdent aucun. Le degré de conversion est limité pour les composites de restauration et le plus souvent proche de $50 \%$, les adhésifs amélo-dentinaires peuvent présenter des degrés de conversion beaucoup plus élevés, de 70 à $85 \%$.

Les spectres Raman ont été obtenus par l'intermédiaire d'un microscope confocal Raman WITec ${ }^{\circledR}$ Alpha 300R (Witec Gmbh, Ulm, Allemagne) (fig. 4). La résolution optique théorique est limitée à $200 \mathrm{~nm}$ latéralement et à $500 \mathrm{~nm}$ verticalement. La résolution spectrale est inférieure à $4 \mathrm{~cm}^{-1}$. La source d'excitation est un laser He-Ne d'une longueur d'onde de $632,8 \mathrm{~nm}$ et d'une puissance de sortie de $35 \mathrm{~mW}$. Le diamètre du sténopé (trou confocal) est de 50 micromètres. Un objectif x 20 avec une ouverture numérique de 0,46 a été utilisé. La grille du spectromètre est réglée à 600 lignes par millimètre.

Le contrôle du microscope Raman s'effectue par le logiciel Witec ${ }^{\circledR}$ dédié. Les spectres ont 


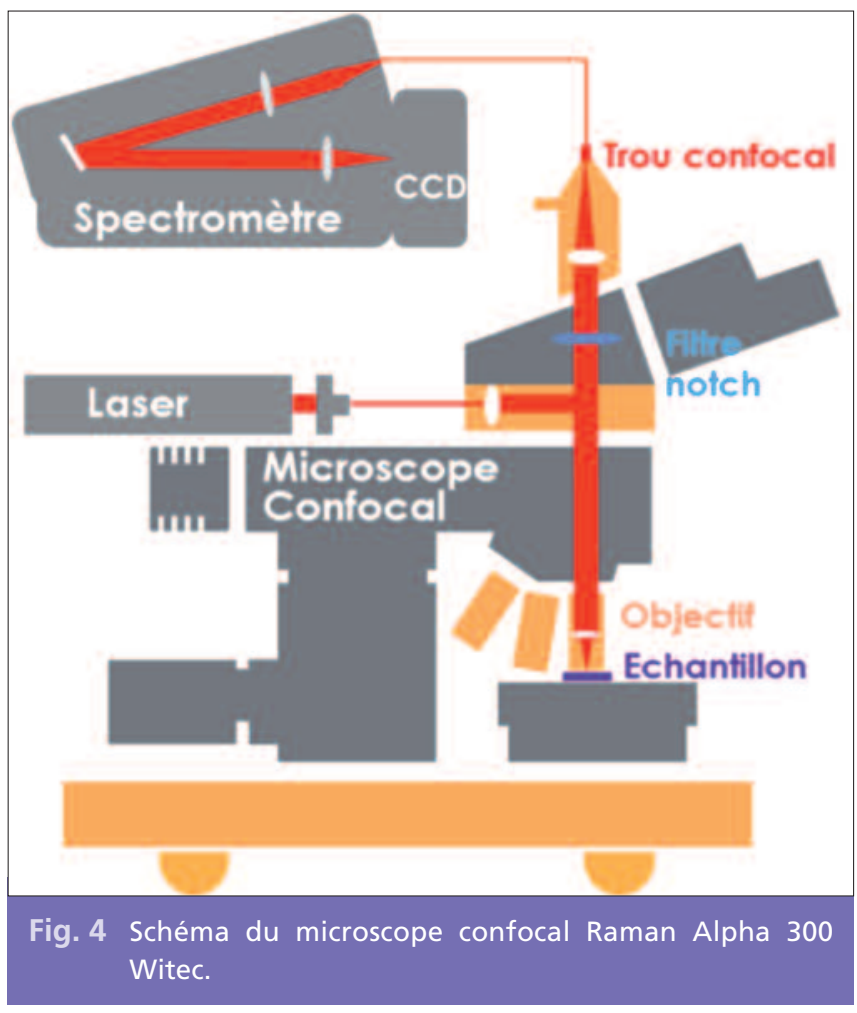

été acquis en centrant la longueur d'onde sur $2000 \mathrm{~cm}^{-1}$, permettant d'obtenir une gamme spectrale comprise entre 600 et $3200 \mathrm{~cm}^{-1}$.

Le matériau testé est le Bonding (agent de couplage) de l'adhésif Scotchbond ${ }^{\mathrm{TM}}$ Multipurpose. C'est un adhésif de $4^{\mathrm{e}}$ génération (de type MR3) de référence, très utilisé dans la littérature pour les tests en laboratoire et les études cliniques. Cet adhésif est adapté à des mesures par spectroscopie Raman, car il présente une fluorescence presque nulle sous irradiation par le laser $632 \mathrm{~nm}$. Sa composition comprend 60 à $70 \%$ de monomères BisGMA et 30 à $40 \%$ de monomères HEMA.

L'insolation a été réalisée avec une lampe Bluephase 16i (Ivoclar Vivadent) de puissance $1600 \mathrm{~mW} / \mathrm{cm}^{2}$ avec un embout de $11,2 \mathrm{~mm}$ de diamètre à une distance de $4 \mathrm{~mm}$, en utilisant le programme haute puissance ( $\mathrm{HI}$ P) pendant 10 secondes.

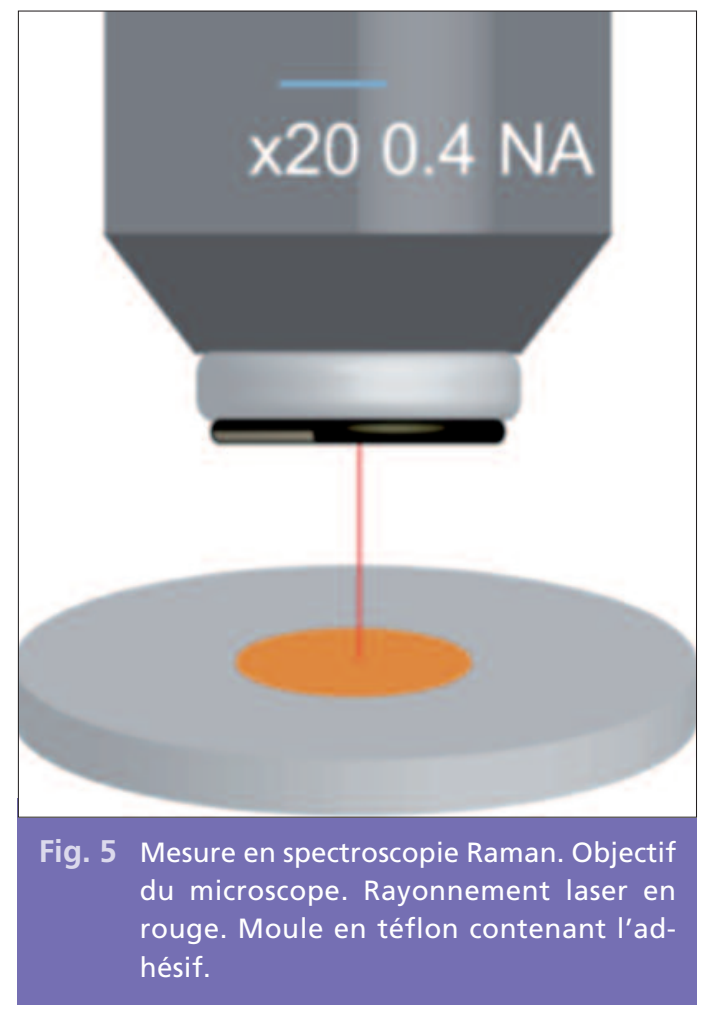

L'adhésif est déposé dans un moule en téflon, placé sous l'objectif du microscope optique sur une tablette piézoélectrique 3 axes contrôlée par l'ordinateur, et le laser est focalisé sur la surface de l'échantillon (fig. 5). La mesure s'effectue en focalisant sur la surface de l'adhésif avant et après polymérisation.

La position du spectre collecté peut être précisément contrôlée en trois dimensions. Pour chaque spectre de référence collecté, le temps d'acquisition choisi est de 1 seconde avec une accumulation de 20 spectres. Pour chaque échantillon, trois mesures sont réalisées sur des zones aléatoires de la surface de l'échantillon avant et après polymérisation au contact de l'air. Pour la comparaison entre les spectres réalisés avant et après polymérisation, une normalisation entre les pics a été réalisée dans une gamme spectrale comprise entre 1500 et $1800 \mathrm{~cm}^{-1}$. 
Pour le calcul du degré de conversion, la hauteur des pics à $1610 \mathrm{~cm}^{-1}$ et à $1640 \mathrm{~cm}^{-1}$ est mesurée, ce qui nous donne l'intensité de chaque pic dans la gamme spectrale normalisée comprise entre 1500 et $1800 \mathrm{~cm}^{-1}$.

Le calcul du degré de conversion est obtenu par la formule :

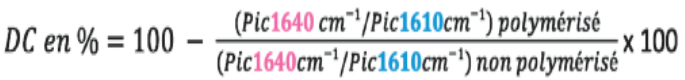

Pour Scotchbond ${ }^{\mathrm{TM}}$ MP adhésif, des tests ont été réalisés en polymérisant le matériau sous une bande de mylar. Ces échantillons peuvent être considérés comme des témoins positifs visà-vis de la polymérisation. La comparaison entre les deux modes de polymérisation avec l'échantillon non polymérisé comme témoin, démontre bien la présence d'une couche d'inhibition (fig. 6 à 8 ).

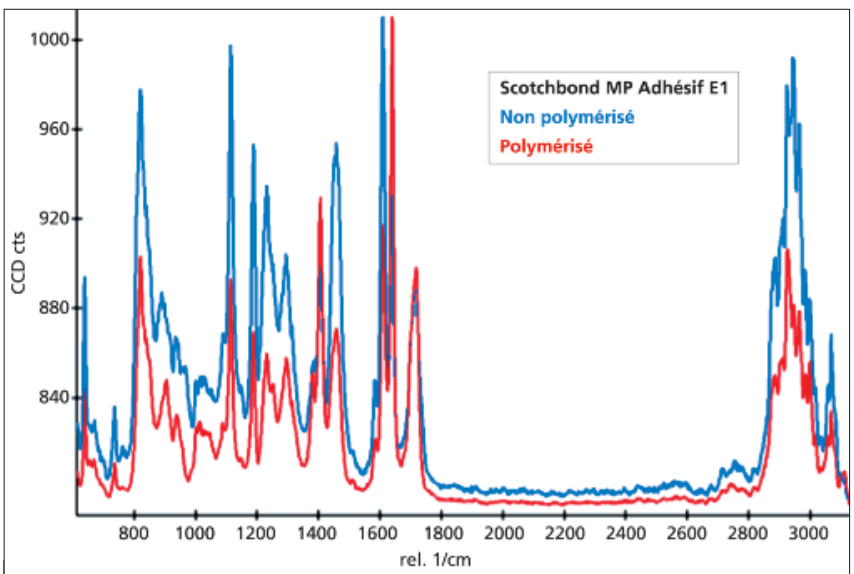

Fig. 6 Spectres Raman de Scotchbond ${ }^{\mathrm{TM}}$ MP Bonding, échantillon E1 non polymérisé (bleu) vs polymérisé (rouge). Objectif x 20. Insolation du matériau au contact de l'air.

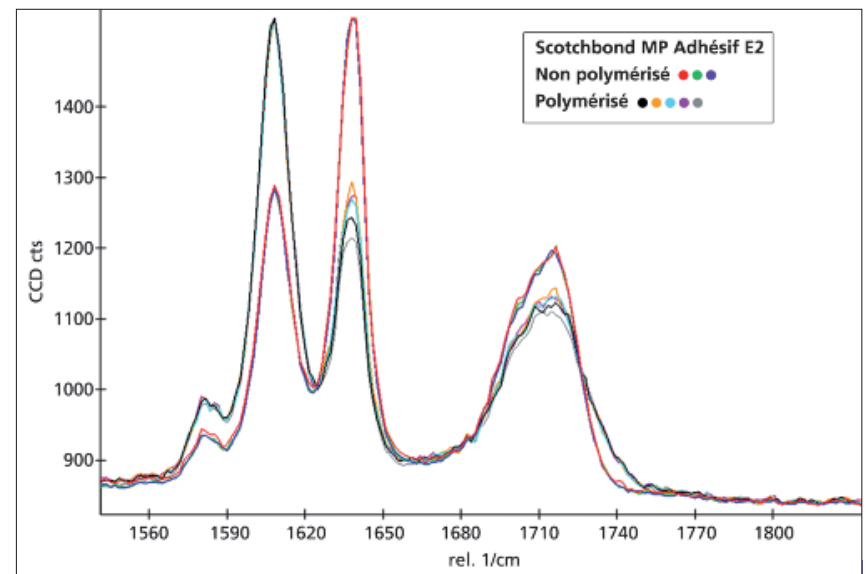

Fig. 7 Spectres Raman, dans la gamme 1500-1800 $\mathrm{cm}^{-1}$, de Scotchbond ${ }^{\mathrm{TM}}$ MP Bonding échantillon E2 non polymérisé (3 zones) vs polymérisé (5 zones). Objectif x 20 . Insolation du matériau au contact de l'air.

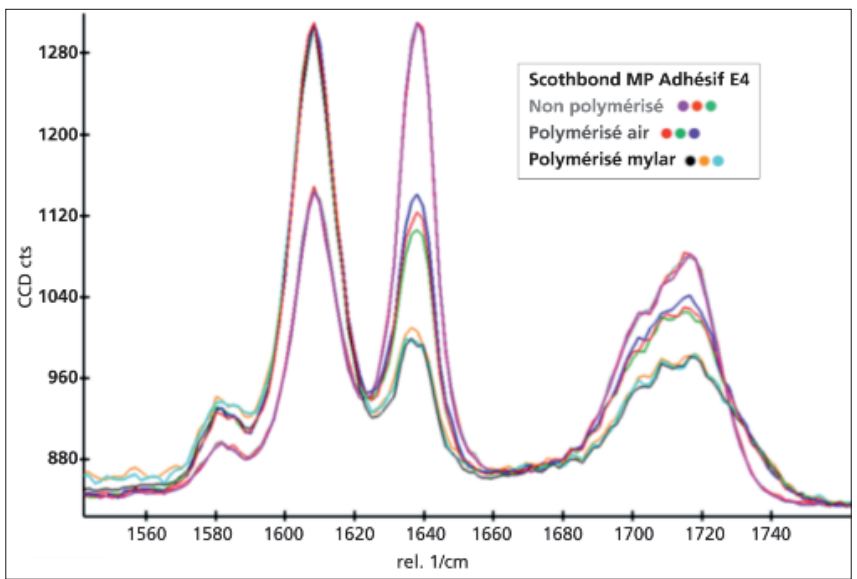

Fig. 8 Spectres Raman de Scotchbond ${ }^{\mathrm{TM}}$ MP Bonding, échantillon E4 non polymérisé 3 zones ( $Z 1$ Z2 Z3) vs polymérisé air 3 zones (Z4 Z5 Z6) vs polymérisé sous Mylar 3 zones (Z7 Z8 Z9). Objectif x 20. Insolation du matériau au contact de l'air et sous Mylar. 


\section{Perspectives cliniques de la spectroscopie Raman}

La spectroscopie Raman est susceptible de devenir un outil diagnostique pour les cancers de la région de la tête et du cou [33] Le diagnostic pourrait être réalisé en temps réel et sans nécessité de prélèvement [34].

En odontologie, la spectroscopie Raman s'envisage comme technique prometteuse de dia- gnostic microbiologique au fauteuil des bactéries orales avec une grande spécificité [35].

Une autre application concerne la détection des caries à un stade précoce [36], sa transposition au fauteuil est en cours de réalisation avec la mise au point de réseaux de guide d'ondes adaptés à cette technologie [37].

\section{Bibliographie}

1. Sutherland SE.

Les assises de la dentisterie fondée sur les faits.

J Can Dent Assoc 2000;66:241-4.

2. Seif J, Vergnes JN, Attal JP. Evidence based dentistry. Définitions actuelles. Inf Dent 2010;20:26-9.

3. Rapport Gaillard 2011. Mission sur l'évolution du statut hospitalo-universitaire. Remis le mardi 3 mai 2011 à Madame Valérie Pécresse, Ministre de l'Enseignement supérieur et de la Recherche par Raphaël Gaillard.

4. JORF 2011.

Arrêté du 17 novembre 2011 relatif à l'organisation et au programme du concours d'internat en odontologie. Journal Officiel $n^{\circ} 275$ du 27 novembre 2011.

5. Bréchot $\mathrm{C}$.

La recherche translationnelle en santé, un nouveau paradigme.
$\mathrm{M} / \mathrm{S}:$ Med Sci

2004;20(10):939-40.

6. Plan cancer 2009-2013. Soutien pour la formation à la recherche translationnelle en cancérologie. Appel à candidatures 2012.

7. Raman CV, Krishnan KS. A new type of secondary radiation. Nature 1928;121(3048):501.

8. Karan K, Yao X, Xu C, Wang $Y$.

Chemical profile of the dentin substrate in non-carious cervical lesions. Dent Mater 2009;25(10):1205-12.

9. Berger SB, Cavalli V, Martin AA,

Soares LE, Arruda MA, Brancalion ML, Giannini M. Effects of combined use of light irradiation and 35\% hydrogen peroxide for dental bleaching on human enamel mineral content.
Photomed Laser Surg 2010;28(4):533-8.

10. Christofferson J, Maize $K$, Ezzahri Y, Shabani J, Wang $X$, Shakouri A. Microscale and nanoscale thermal characterization techniques. J Electron Packag 2008;130(4):41101-6.

11. Coopera CA, Younga RJ, Halsallb M.

Investigation into the deformation of carbon nanotubes and their composites through the use of Raman spectroscopy. Composites. Part A, Applied science and manufacturing 2001;32(3-4):401-11.

12. O'Shea DC, Bartlett ML, Young RA.

Compositional analysis of apatites with laser-raman spectroscopy:

(oh,f,cl) apatites.

Arch Oral Biol

1974;19(11):995-1006. 
13. Tsuda H, Arends J. Raman spectroscopy in dental research: a short review of recent studies. Adv Dent Res 1997;11(4):539-47.

14. Suzuki M, Kato $H$, Wakumoto S. Vibrational analysis by Raman spectroscopy of the interface between dental adhesive resin and dentin. J Dent Res 1991;70(7):1092-7.

15. Wang $Y$, Spencer $P$. Quantifying adhesive penetration in adhesive/dentin interface using confocal Raman microspectroscopy. J Biomed Mater Res 2002;59(1):46-55.

16 Aminzadeh A, Shahabi S, Walsh LJ.

Raman spectroscopic studies of $\mathrm{CO} 2$ laser-irradiated human dental enamel. Spectrochim Acta A Mol Biomol Spectrosc 1999;55A(6):1303-8.

17. Kwon $\mathrm{YH}, \mathrm{Kwon}$ OW, Kim HI, Kim KH. Nd:YAG laser ablation and acid resistance of enamel. Dent Mater J 2003;22(3):404-11.

18. Park HJ, Kwon TY, Nam SH, Kim HJ, Kim KH, Kim YJ. Changes in bovine enamel after treatment with a $30 \%$ hydrogen peroxide bleaching agent. Dent Mater J 2004;23(4):517-21.

19 Xu B, Li Q, Wang Y. Effects of $\mathrm{pH}$ values of hydrogen peroxide bleaching agents on enamel surface properties.

Oper Dent 2011;36(5):554-62.

20. Sato $H$, Yamada $K$, Pezzotti G, Nawa M, Ban S. Mechanical properties of dental zirconia ceramics changed with sandblasting and heat treatment.

Dent Mater J 2008;27(3):408-14.

21. Presser V, Keuper M, Berthold C, Nickel KG.

Experimental determination of the Raman sampling depth in zirconia ceramics. Appl Spectrosc 2009;63(11):1288-92.

22. Shin WS, Li XF, Schwartz B, Wunder SL, Baran GR. Determination of the degree of cure of dental resins using Raman and FT-Raman spectroscopy.

Dent Mater 1993;9(5):317-24.

23. Miletic V, Santini A. Micro-Raman spectroscopic analysis of the degree of conversion of composite resins containing different initiators cured by polywave or monowave LED units.

J Dent 2012;40(2):106-13.

24. Navarra CO, Cadenaro $M$, Codan B, Mazzoni A, Sergo $V$

De Stefano Dorigo E, Breschi L.

Degree of conversion and interfacial nanoleakage expression of three one-step self-etch adhesives.

Eur J Oral Sci 2009;117(4):463-9.

25 Costa SXS, Galvão MR, Jacomassi DP, Bernardi MIB, Hernandes AC, de Souza Rastelli AN, Andrade MF.
Continuous and gradual photo-activation methods: influence on degree of conversion and crosslink density of composite resins.. J Therm Anal Cal 2011;103(1):219-27.

26. Kanehira $M$, Finger $W J$, Hoffmann M, Endo T,

Komatsu M.

Relationship between degree of polymerization and enamel bonding strength with self-etching adhesives. J Adhes Dent 2006;8(4)211-6.

27. Sadek FT, Calheiros FC, Cardoso PE, Kawano Y, Tay F, Ferrari M.

Early and 24-hour bond strength and degree of conversion of etch-and-rinse and self-etch adhesives. Am J Dent 2008;21(1):30-4.

28. Fujita K, Ikemi T, Nishiyama N.

Effects of particle size of silica filler on polymerization conversion in a light-curing resin composite.

Dent Mater 2011;27(11):1079-85.

29. Owens BM.

Evaluation of curing performance of light-emitting polymerization units. Gen Dent 2006;54(1):17-20.

30. Vallittu PK. Unpolymerized surface layer of autopolymerizing polymethyl methacrylate resin.

J Oral Rehabil

1999;26(3):208-12. 
31. Gauthier MA, Stangel I, Ellis TH, Zhu XX. Oxygen inhibition in dental resins. J Dent Res 2005;84(8):725-9.

32. Truffier-Boutry D, Place $E$, Devaux J, Leloup G. Interfacial layer characterization in dental composite. J Oral Rehabil 2003;30(1):74-7.

33. Harris AT, Rennie A, Waqar-Uddin $\mathrm{H}$, Wheatley SR, Ghosh SK, Martin-Hirsch DP, Fisher SE, High AS, Kirkham J, Upile T.
Raman spectroscopy in head and neck cancer. Head Neck Oncol 2010;5(2):26.

34. Swinson $B$, Jerjes $W$, El-Maaytah M, Norris P, Hopper C. Optical techniques in diagnosis of head and neck malignancy. Oral Oncol 2006;42(3):221-8.

35. Howell SC, Haffajee AD, Pagonis TC, Guze KA. Laser raman spectroscopy as a potential chair-side microbiological diagnostic device.
J Endod 2011;37(7):968-72.

36. Choo-Smith LP, Dong CC, Cleghorn B, Hewko M. Shedding new light on early caries detection. J Can Dent Assoc 2008;74(10):913-8.

37. Ismail N, Choo-Smith LP, Wörhoff K, Driessen A, Baclig AC, Caspers PJ, Puppels GJ, de Ridder RM, Pollnau M.

Raman spectroscopy with an integrated arrayed-waveguide grating. Opt Lett 2011;36(23):4629-31.

\section{SUMMARY}

\section{Confocal Raman spectroscopy in dentistry. From basic research to clinical applications}

Bruno JACQUOT, Jean-Cédric DURAND, Hugues FOUQUET, Hamideh SALEHI, Perrine MARGUET-GAUDIN, Bernard LEVALLOIS, Frédéric CUISINIER

\section{Keywords \\ - Raman spectroscopy - basic research - degree of conversion - composite resins - oxygen inhibited layer}

We present a new tool for observing surfaces, used in basic research, the Raman spectroscopy. This technique offers numerous possibilities in the dental tissues and materials analysis and imaging.

The Raman effect was discovered by Chandrashekhara Venkata Raman in 1928.

Applications are very diverse in Dentistry, but Raman spectroscopy is essentially utilized to analyze the degree of conversion of composite resins directly, or within the hybrid layer for dental adhesives.

Raman spectroscopy, such as Infrared spectroscopy, is a vibrational method, which allows a direct approach to the degree of conversion and quantification of the thickness of the oxygen-inhibited layer. Various applications are illustrated with the measurement of the degree of conversion of a dentin bonding agent and the determination of its oxygen-inhibited layer. 